J. Lake Sci. (湖泊科学), 2015, 27(4):607-615

DOI $10.18307 / 2015.0407$

(c) 2015 by Journal of Lake Sciences

\title{
表层底泥翻耕对太湖藻源性湖泛的预控作用
}

\author{
何 伟 $^{1}$, 陈显权 ${ }^{1}$, 顾春新 ${ }^{2}$, 范成新 ${ }^{3 * *}$, , 蔡丽婧 $^{1}$ \\ ( 1 : 上海勘测设计研究院有限公司, 上海 200434) \\ （2:无锡市锡山区水利局,无锡 214101) \\ (3: 中国科学院南京地理与湖泊研究所, 南京 210008)
}

\begin{abstract}
摘 要: 表层底泥已被证实是引发或诱发太湖湖泛发生的主要因素,但能否通过像控制湖泊内源污染的翻耕方式对湖泛 形成预控,则有待与过程有关的实验研究. 模拟研究太湖易发湖泛区底泥翻耕对藻源性湖泛的控制作用,跟踪监测不同 翻耕深度和翻耕后时长,对照和处理组上覆水的视觉、嗅觉、营养盐和挥发性硫化物浓度, 以及表层底泥理化性质的变 化. 结果表明,未翻耕的对照组在模拟实验中发生了湖泛,翻耕 $10 \mathrm{~cm}$ 和翻耕 $20 \mathrm{~cm}$ 差异不大,但翻耕后时长对湖泛发生 影响较大. 刚翻耕后 (1 d) 即进行藻源性湖泛模拟的处理组发生了湖泛,但在程度和持续时间上均低于对照组;翻耕 $180 \mathrm{~d}$ 和 $540 \mathrm{~d}$ 的处理组, $10 \mathrm{~cm}$ 和 $20 \mathrm{~cm}$ 翻耕深度均未发生湖泛. 合理选择翻耕的施工时间, 可以有效预防和控制湖泛的发生.
\end{abstract}

关键词: 表层底泥; 翻耕模拟; 湖泛;理化性质;挥发性硫化物

\section{Effects of plow-tillage on the prevention and control of algae-caused black bloom}

\author{
HE Wei ${ }^{1}$, CHEN Yuquan ${ }^{1}$, GU Chunxin ${ }^{2}$, FAN Chengxin ${ }^{3} \&$ CAI Lijing ${ }^{1}$ \\ (1: Shanghai Investigation, Design \& Research Institute Co. , Ltd., Shanghai 200434, P. R. China) \\ (2: Wuxi Xishan District Water Resources Bureau, Wuxi 214101, P. R. China) \\ (3: Nanjing Institute of Geography and Limnology, Chinese Academy of Sciences, Nanjing 210008, P. R. China)
}

\begin{abstract}
Black-bloom was an environment issue that often occurred recently and the polluted surface sediment was known as the most important reason for its occurrence. Plow-tillage was a cost-effective and practicable engineering measure which was designed for internal loading control. Based on the simulated experiment in laboratory, effects of plow-tillage on the prevention and control of algae-caused black bloom was studied. The color and odor, nutrient concentration of overlaying water, the concentrations of volatile sulfur compounds and physicochemical characteristic of sediment were measured. The results were showed as follows: black bloom occurred in both control check set and treatment sets l day after plow-tillage for the two diffirent plow-tillage depths, but its duration and degree in control check set were more serious than those in treatment sets 1 day after plow-tillage. The treatment sets of 180 and 540 days after plow-tillage of both depths didn’t experienced black bloom during the whole simulated experiment. Overall, the appropriate project time of plow-tillage can prevent the occurrence and control the degree of algae-caused black bloom.
\end{abstract}

Keywords: Surface sediment; plow-tillage; black bloom; physicochemical properties; volatile sulfur compounds

湖泛 (black bloom) 又叫黑水团或污水团,是近年来在太湖、巢湖、滇池等湖泊频繁发生的一种水体严重 污染现象. 在太湖等长江中下游湖泊,湖泛主要是由水华蓝藻聚集、死亡分解引起的,因此也称为藻源性湖 泛.湖泛最典型的感官特征就是视觉上的“黑”和嗅觉上的“臭”,同时伴有溶解氧浓度极低(甚至出现完全 厌氧），化学耗氧量、氨氮、硫化物和二甲基硫梄类等物质浓度高的特点 ${ }^{[1]} .2007$ 年 5 月 29 日, 太湖无锡贡湖 水厂南泉取水口发生湖泛, 造成水厂停产、近 200 万居民饮用水短缺的严重事件,引起国内外的广泛关

* 江苏省太湖水污染治理专项 ( TH2013214)、中国科学院南京地理与湖泊研究所 “一三五” 战略发展规划项目 ( NIGLAS2012135008) 和国家自然科学基金项目 (41103033) 联合资助. 2014-06-06 收稿;2014-09-22 收修改 稿. 何伟(1985 ), 男,博士;E-mail:hewei1022@ gmail.com.

** 通信作者;E-mail:cxfan@ niglas. ac. cn. 
注 $^{[1-3]}$. 相关研究表明, 湖泛水体的黑色是由硫化物与底泥中的金属离子结合形成的金属硫化物 ${ }^{[4-5]}$, 其臭味 主要是由二甲基三硫醚等挥发性硫化物产生的 ${ }^{[1,2,6]}$.

Yang 等 ${ }^{[2]}$ 认为湖泛氨氮浓度的增加幅度超过了仅由藻华发生而引起氨氮浓度的增加幅度. 孔繁翔等 ${ }^{[7]}$ 通过对 2007 年太湖湖泛成因进行分析, 认为湖泛是由于蓝藻水华淤积在沉积物上, 厌氧降解形成溶解性有 机物释放进人水体而形成的. 盛东等 ${ }^{[4]}$ 通过实验证明仅有藻类无法形成湖泛, 需有污染沉积物的参与. 陆 桂华等 ${ }^{8}{ }^{[}$通过对太湖全湖的巡查认为湖泛主要发生在湖泊淤泥和流泥分布较厚、藻类易聚积的区域. 因 此目前预防湖泛的技术手段中, 除了控制湖泊外源污染物的输人, 移除蓝藻以防止其大量堆积外, 最有效 的手段就是将富含营养盐和有机物的表层沉积物通过工程技术手段进行移除 ${ }^{[7-8]}$. 通过室内模拟实验, 认 为对太湖月亮湾、南泉水厂和符渎港等区域表层污染严重的沉积物进行疏浚后, 可以有效控制湖泛的发 生和程度 ${ }^{[9]}$.

底泥翻耕是一种借用机械设备, 改变或颠覆水下底质性质和物理位置的作业方法, 常用于受海洋养殖 污染影响的滨岸带水质改善 ${ }^{[10-11]}$. 经对太湖沉积物的模拟, 认为底泥翻耕也可以高效地治理湖泊氮、磷内

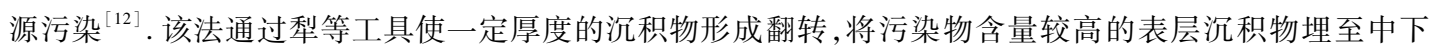
部, 而将较为清洁的下部沉积物翻转至表层, 一方面可明显改善表层沉积物质量, 抑制其对水底环境的不 良影响; 另一方面重建沉积物一水界面, 达到控制污染物向上覆水释放等目的. 古小治等对南四湖沉积物改 良的研究表明, 对沉积物的耕作能够有效地改善沉积物的物理结构及氧气的垂向分布, 实现原位处理且花 费少 ${ }^{[13-14]}$.

目前针对湖泛的研究主要集中在野外跟踪监测和成因推测上 ${ }^{[2,3,15]}$, 由于湖泛暴发的随机性和湖泛样 品的不稳定性, 难以对湖泛进行深人研究. 因此, 采用室内模拟手段, 再现藻源性湖泛, 对湖泛的发生过程及 其机理研究有着非常重要的现实意义 ${ }^{[5,8,16-17]}$. 现有防治湖泛的工程措施花费大, 难以大面积施工, 而翻耕作 为一种有效控制沉积物内源污染的方式, 具有操作简单、易实施、成本低的优点, 但是在控制湖泛的效果上 尚无相关研究. 因此本文通过实验室模拟太湖湖泛发生的自然条件, 研究沉积物不同翻耕深度和翻耕后的 时长对湖泛发生、发展过程控制或减缓作用, 以期更好地为预防太湖藻源性湖泛的发生提供新的技术手段 和理论依据.

\section{1 材料与方法}

\section{1 研究点概况}

竺山湾位于太湖西北部, 北起百渎口, 南至马山咀一线, 面积 $57.2 \mathrm{~km}^{2}$, 该湖区水体多受西部太滆运河、 殷村港等河道污水汇人影响, 水体富营养化程度高, 已是太湖水质污染最严重且恶化速率最快的湖湾之

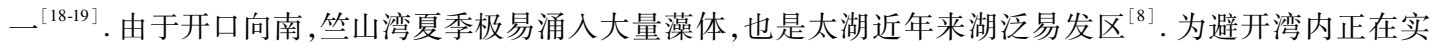
施或计划进行生态疏浚的区域, 将沉积物样品采样点设定在竺山湾湾口处 $\left(31.39^{\circ} \mathrm{N}, 120.05^{\circ} \mathrm{E}\right)$.

\section{2 样品的采集及处理}

采用大口径柱状采样器 ( $\Phi 110 \mathrm{~mm}, \mathrm{~L} 500 \mathrm{~mm}$ Rigo Co., Japan) 进行采样, 采集深度大于 $30 \mathrm{~cm}$ 的沉积物 柱状样; 利用采水器采集采样点湖水用于模拟实验; 利用浮游生物网于竺山湾湖区采集藻类, 用于实验中人 为投加藻类模拟藻华 ${ }^{[5,16-17]}$.

沉积物处理共设置翻耕 $10 \mathrm{~cm}$ ( PT-10) 和翻耕 $20 \mathrm{~cm}$ ( PT-20) 两组深度. 以翻耕 $10 \mathrm{~cm}$ 为例, 利用一个长 $10 \mathrm{~cm}$ 的有机玻璃管, 将表层 $10 \mathrm{~cm}$ 厚的沉积物翻转, 翻转后的沉积物移到原柱状样中相应深度处, 再用输 液用点滴软管无扰动地加人过滤后的湖水. 经过翻耕处理的沉积物柱样, 原先营养盐含量高的表层沉积物 被覆盖在设计的翻耕深度处, 原先翻耕深度处营养盐含量较低的底层沉积物被翻耕到沉积物表面, 与上覆 水共同作用形成新的沉积物一水界面.

制备的沉积物柱样上端敞口, 沉积物部分用铝簿包裹避光, 在水桶中培养, 桶内水位高出柱样 $15 \sim$ $20 \mathrm{~cm}$, 按照采样点的水温进行培养 ( 误差 $\pm 2^{\circ} \mathrm{C}$ ), 水桶中间歇曝气, 曝气头位于桶底处, 培养使用过滤藻类 后的湖水, 每 2 周更换 1 次. 样品中对照和翻耕后 $1 \mathrm{~d}$ 的两个处理水平在水桶中培养 $3 \mathrm{~d}$, 而翻耕后 180 和 $540 \mathrm{~d}$ 的样品均在翻耕后在水桶中培养, 分别培养 180 和 $540 \mathrm{~d}$. 


\section{3 实验方法}

实验共设计 7 根柱状样, 分别为对照 (CK)、翻耕后 $1 \mathrm{~d}$ ( PT-10-d1、PT-20-d1)、翻耕后 $180 \mathrm{~d}(\mathrm{PT}-10-\mathrm{d} 180$ 、 PT-20-d180)、翻耕后 540 d( PT-10-d540、PT-20-d540).

实验所用柱状样长约 $2 \mathrm{~m}$, 沉积物高 $35 \sim 40 \mathrm{~cm}$, 模拟水深为 $160 \mathrm{~cm}$, 在实验的第 $2 \mathrm{~d}$ 称取沥干水分的藻 体 $30.50 \mathrm{~g}$ (约 $\left.5000 \mathrm{~g} / \mathrm{m}^{2}\right)^{[5,16-17]}$ 加人各柱样中, 环境温度控制为 $25 \pm 1^{\circ} \mathrm{C}$, 模拟 2008 年 5 月湖泛时太湖的平 均气温, 实验在自然光照条件下进行, 底部管体用铝箔包裹以避光.

模拟实验为 7 根柱样同步进行, 每天在沉积物一水界面上 $20 \mathrm{~cm}$ 处采集 $75 \mathrm{ml}$ 水样, 同时补人相应体积 的湖水,并在计算上覆水营养盐浓度时进行校正. 其中 $50 \mathrm{ml}$ 水样过滤后 (GF/C, Whatman) 用于分析铵态氮 $\left(\mathrm{NH}_{4}^{+}-\mathrm{N}\right)$ 、磷酸盐 $\left(\mathrm{PO}_{4}^{3-}-\mathrm{P}\right)$ 浓度. $25 \mathrm{ml}$ 水样装人萃取瓶中冷冻保存, 实验结束后统一测定气相指标. 每天 在采集水样的同时对各个沉积物柱样上覆水的溶氧含量进行测定和记录.

沉积物在模拟实验之后按 $0 \sim 1 、 1 \sim 2 、 2 \sim 3 、 3 \sim 4 、 4 \sim 5$ 和 $5 \sim 6 \mathrm{~cm}$ 的厚度将上层沉积物分层, 一部分鲜 样迅速用氮气排空的密封袋保存, 用于分析含水率; 剩下的样品经冷冻干燥机冻干之后, 研磨, 过 100 目篮, 分析沉积物的烧失重 ( LOI) 、总磷 (TP) 和总氮 (TN).

\section{4 样品分析}

1.4.1 水体的视觉和嗅觉 上覆水中水色和嗅味上均出现明显变化的柱状样定义为湖泛的发生. 为了 对模拟实验的过程进行描述, 将实验中水体呈现的颜色和气味均人为定义为 4 个等级 ${ }^{[9,20]}$, 水色: 无 色、灰色、浅黑、黑色; 嗅味: 无味、轻微、中度、重度. 分别以 $0 、 1 、 2 、 3$ 代表水色和嗅味上由轻到重的 4 个等级程度.

1.4.2 水体中致臭物质 挥发性硫化物包括挥发性无机硫化物硫化氢 $\left(\mathrm{H}_{2} \mathrm{~S}\right)$ 和挥发性有机硫化物甲硫醇 (MeSH)、甲硫醚 (DMS) 、二甲基二硫醚 (DMDS) 和二甲基三硫醚 (DMTS), 挥发性硫化物通常具有强烈的恶 臭气味, 嗅阈值低, 即使在较低浓度时也会对水或食物的味道产生很大影响. 因此对上覆水中上述 5 种物质 进行分析测定.

利用顶空固相微萃取法 (HS-SPME) 对挥发性硫化物进行富集, 然后与 Agilent 7890A GC-FPD (Agilent, USA) 系统组合完成对挥发性硫化物浓度的测定.

顶空固相微萃取手柄和萃取头购自 Supelco 公司, 萃取头表面的吸附涂层为 CAR/PDMS (75 $\mu \mathrm{m}$, No. 57318 , USA ), Agilent 7890A GC-FPD 气相色谱一火焰光度检测器, 惰性化进样口, 色谱柱为 Gas pro 毛细管柱 $(60 \mathrm{~m} \times 0.32 \mathrm{~mm})$, 棕色玻璃萃取瓶的容积为 $40 \mathrm{ml}$, 与 PTFE 涂层硅胶垫配合使用.

顶空固相微萃取的温度为 $65^{\circ} \mathrm{C}$, 搅拌速率为 500 转 $/ \mathrm{min}$, 萃取时间为 $30 \mathrm{~min}$. GC-FDA 载气为高纯氦气, 流量为 $3 \mathrm{ml} / \mathrm{min}$, 氢气流量为 $50 \mathrm{ml} / \mathrm{min}$, 空气流量为 $65 \mathrm{ml} / \mathrm{min}^{[21-22]}$.

1.4.3 理化指标 水样中的 $\mathrm{NH}_{4}^{+}-\mathrm{N}$ 浓度利用纳氏试剂法通过分光光度计测定 ${ }^{[23]}, \mathrm{PO}_{4}^{3-}-\mathrm{P}$ 浓度利用磷钼蓝 法通过分光光度计测定 ${ }^{[24]}$. 沉积物的含水率利用烘干法来测定; 沉积物的有机质含量用 LOI 来代替 ${ }^{[25]}$; 沉 积物 TP 含量在消解后利用锄锑抗分光光度法测定 ${ }^{[26]}$; 沉积物 TN 含量用过硫酸钾消解后紫外分光光度法 测定 ${ }^{[23]}$.

\section{2 结果与讨论}

实验持续 $22 \mathrm{~d}$, 在整个实验周期中, 7 根沉积物柱状样品中共有 3 根柱样出现湖泛特征, 即上覆水长时 间发黑发臭, 根据以往湖泛室内模拟实验,结合后期水体挥发性硫化物和理化指标的检测, 认为这 3 根柱状 样发生了湖泛,分别是柱样 CK、PT-10-d1 和 PT-20-d1.

\section{1 水体视觉和嗅觉的变化}

在第 $12 \mathrm{~d}$ 时柱样 CK 和 PT-10-d1 柱样上覆水颜色开始发灰, $1 \mathrm{~d}$ 后 PT-20-d1 柱样也出现了水色的变化, 与此同时, CK 组水色变深,呈浅黑色,在第 $14 \mathrm{~d}$ 时, CK 组水色继续加深,呈黑色,持续 $2 \mathrm{~d}$ 后 $\mathrm{CK}$ 组上覆水的 颜色开始变浅,至第 $22 \mathrm{~d}$ 水色恢复为无色. 而翻耕 $1 \mathrm{~d}$ 后的 2 根沉积物柱样 (PT-10-d 1 、PT-20-d 1 ) 在上覆水 水体颜色上要明显浅于 CK 组,在整个实验周期中, 这 2 根沉积物柱样上覆水的水色在发黑之后均保持在灰 色和浅黑色,没有变成黑色,说明在色度指标上,这 2 根沉积物柱样要优于 CK 组 (表 1 ). 
表 1 湖泛模拟实验中上覆水体的水色变化

Tab. 1 Changes in water color during the experiment

\begin{tabular}{|c|c|c|c|c|c|c|c|c|c|c|c|c|c|c|c|c|c|c|c|c|c|c|}
\hline \multirow{2}{*}{ 实验组 } & \multicolumn{22}{|c|}{ 时间/d } \\
\hline & 1 & 2 & 3 & 4 & 5 & 6 & 7 & 8 & 9 & 10 & 11 & 12 & 13 & 14 & 15 & 16 & 17 & 18 & 19 & 20 & 21 & 22 \\
\hline CK & 0 & 0 & 0 & 0 & 0 & 0 & 0 & 0 & 0 & 0 & 0 & 1 & 2 & 3 & 3 & 2 & 2 & 2 & 2 & 1 & 1 & 0 \\
\hline PT-10-d1 & 0 & 0 & 0 & 0 & 0 & 0 & 0 & 0 & 0 & 0 & 0 & 1 & 1 & 2 & 1 & 1 & 1 & 2 & 2 & 1 & 0 & 0 \\
\hline PT-20-d1 & 0 & 0 & 0 & 0 & 0 & 0 & 0 & 0 & 0 & 0 & 0 & 0 & 1 & 1 & 2 & 1 & 2 & 2 & 1 & 2 & 1 & 0 \\
\hline PT-10-d180 & 0 & 0 & 0 & 0 & 0 & 0 & 0 & 0 & 0 & 0 & 0 & 0 & 0 & 0 & 0 & 0 & 0 & 0 & 1 & 0 & 0 & 0 \\
\hline PT-20-d180 & 0 & 0 & 0 & 0 & 0 & 0 & 0 & 0 & 0 & 0 & 0 & 0 & 0 & 0 & 0 & 0 & 0 & 0 & 0 & 0 & 0 & 0 \\
\hline PT-10-d540 & 0 & 0 & 0 & 0 & 0 & 0 & 0 & 0 & 0 & 0 & 0 & 0 & 0 & 0 & 0 & 0 & 0 & 0 & 0 & 0 & 0 & 0 \\
\hline PT-20-d540 & 0 & 0 & 0 & 0 & 0 & 0 & 0 & 0 & 0 & 0 & 0 & 0 & 0 & 0 & 0 & 0 & 0 & 0 & 0 & 0 & 0 & 0 \\
\hline
\end{tabular}

在嗅觉方面, 发生湖泛的柱样上覆水出现臭味要较视觉变化提前 $1 \mathrm{~d}$, 在第 $11 \mathrm{~d}$ 时 CK 和 PT-20-d 1 柱样 的上覆水便出现臭味, 在第 $12 \mathrm{~d}$, PT-10-d1 柱样也开始出现臭味. CK 和 PT-10-d1 柱样上覆水一直持续到实 验结束时还有轻微臭味, 而最早出现臭味的 PT-10-d1 柱样则在第 $21 \mathrm{~d}$ 时水体中的臭味消失 (表 2).

表 2 湖泛模拟实验中上覆水体的嗅味变化

Tab. 2 Changes in the odor of the water samples during the experiment

\begin{tabular}{|c|c|c|c|c|c|c|c|c|c|c|c|c|c|c|c|c|c|c|c|c|c|c|}
\hline \multirow{2}{*}{ 实验组 } & \multicolumn{22}{|c|}{ 时间/d } \\
\hline & 1 & 2 & 3 & 4 & 5 & 6 & 7 & 8 & 9 & 10 & 11 & 12 & 13 & 14 & 15 & 16 & 17 & 18 & 19 & 20 & 21 & 22 \\
\hline CK & 0 & 0 & 0 & 0 & 0 & 0 & 0 & 0 & 0 & 0 & 1 & 1 & 2 & 3 & 3 & 2 & 2 & 2 & 1 & 1 & 2 & 1 \\
\hline PT-10-d1 & 0 & 0 & 0 & 0 & 0 & 0 & 0 & 0 & 0 & 0 & 0 & 1 & 1 & 2 & 1 & 2 & 1 & 1 & 2 & 1 & 1 & 1 \\
\hline PT-20-d1 & 0 & 0 & 0 & 0 & 0 & 0 & 0 & 0 & 0 & 0 & 1 & 1 & 2 & 1 & 2 & 1 & 1 & 2 & 1 & 1 & 0 & 0 \\
\hline PT-10-d180 & 0 & 0 & 0 & 0 & 0 & 0 & 0 & 0 & 0 & 0 & 0 & 0 & 0 & 0 & 0 & 0 & 0 & 0 & 1 & 0 & 0 & 0 \\
\hline PT-20-d180 & 0 & 0 & 0 & 0 & 0 & 0 & 0 & 0 & 0 & 0 & 0 & 0 & 0 & 0 & 0 & 0 & 0 & 0 & 0 & 0 & 0 & 0 \\
\hline PT-10-d540 & 0 & 0 & 0 & 0 & 0 & 0 & 0 & 0 & 0 & 0 & 0 & 0 & 0 & 0 & 0 & 0 & 0 & 0 & 0 & 0 & 0 & 0 \\
\hline PT-20-d540 & 0 & 0 & 0 & 0 & 0 & 0 & 0 & 0 & 0 & 0 & 0 & 0 & 0 & 0 & 0 & 0 & 0 & 0 & 0 & 0 & 0 & 0 \\
\hline
\end{tabular}

实验中有 3 根柱样发生湖泛, 因此认为翻耕 $1 \mathrm{~d}$ 后的处理组 (PT-10-d 1 PT-20-d1 ) 无论是在水体黑臭程 度上还是持续时间上都要轻于 CK 组. 在目前对湖泛尚无系统和完整的理论性研究的情况下, 水体的视觉和 嗅觉指标虽然直观, 但由于是人为判定, 所以在使用上还需要结合其它指标, 才能做出更加客观的判断.

国外的湖泊和近海中也有类似黑臭水体的报道, 有关美国伊利诺伊州的露天湖矿和 Lower Mystic 湖的 研究表明, 这 2 个湖泊的黑水均为金属硫化物所致 ${ }^{[27-28]}$, 而后者的臭味则是 $\mathrm{H}_{2} \mathrm{~S}$ 从湖中逸散造成的. 通过对 德国 Wadden 海自然发生和模拟的黑水团进行一系列研究, 认为其黑色和臭味是在高有机物负荷下, 硫酸还 原作用在有机物分解中占据优势,生成黑色的硫化物和 $\mathrm{H}_{2} \mathrm{~S}^{[20,29-31]}$.

相关研究表明,太湖藻源性湖泛的黑色物质主要是金属硫化物, 臭味主要是由 DMTS 等挥发性硫化物产 生的 ${ }^{[2]}$. 因此, 在对水色和嗅味做感官记录的同时, 对水体中的挥发性硫化物也进行相应检测, 以做到相互 印证.

\section{2 水体中致臭物质}

实验第 $11 \mathrm{~d}$, 各柱样上覆水中 $\mathrm{H}_{2} \mathrm{~S}$ 浓度开始明显提高, CK、PT-20-d1 和 PT-10-d1 3 根柱样上覆水中 $\mathrm{H}_{2} \mathrm{~S}$ 浓度增长速度最快, 均在第 $14 \mathrm{~d}$ 达到最高, 最大值分别为 $2384.40 、 2017.20$ 和 $1358.55 \mu \mathrm{g} / \mathrm{L}$, 之后开始下 降, 但是在实验后期仍明显高于其余 4 根柱样中的 $\mathrm{H}_{2} \mathrm{~S}$ 浓度 (图 1). 值得注意的是, 在实验第 $9 \mathrm{~d}$, PT-10-d180柱样有轻微的臭味, 而此时该柱样仅 $\mathrm{H}_{2} \mathrm{~S}$ 浓度较高, 因此在湖泛的发生中, 仅 $\mathrm{H}_{2} \mathrm{~S}$ 浓度高于 $900 \mu \mathrm{g} / \mathrm{L}$ 时, 就会产生轻微嗅觉上的不适.

由于实验中投加藻体是在第 $2 \mathrm{~d}$ 开始的, 因此所有柱样上覆水的 MeSH 浓度在第 $2 \mathrm{~d}$ 小幅增长, 但很快 消失. 从第 $6 \mathrm{~d}$ 开始, CK 和 PT-10-d 1 柱样的 MeSH 浓度就开始增加, 其中 CK 柱样在第 $10 \mathrm{~d}$ 的增幅变大. 而 在第 $11 \mathrm{~d}, 7$ 根柱样上覆水的 $\mathrm{MeSH}$ 浓度都有一个爆发式增加的过程, 在接下来的数天中, 7 根柱样上覆水的 
MeSH 浓度走势开始有较大的分化, 其中 CK、PT-10-d1 和PT-20-d1柱样的 MeSH 浓度在第 $11 \mathrm{~d}$ 时增长幅度最 高, 然后保持稳定的趋势, 并在第 $17 \mathrm{~d}$ 开始逐步下降,但是一直到实验结束, 这 3 根沉积物柱样的 MeSH 浓 度还是要高于其它 4 根柱样 ( 图 1).

7 根柱样上覆水中的 DMS 浓度在实验前期处于较低水平, 均在 $1 \mu \mathrm{g} / \mathrm{L}$ 以下, 第 $10 \mathrm{~d}$ 和第 $11 \mathrm{~d}$ 时, 所有 柱样上覆水中的 DMS 浓度均开始大幅上升, CK、PT-10-d1 和 PT-20-d1 柱样的 DMS 浓度大幅增长之后, 是其 余 4 个柱样的 1 倍左右. 第 $17 \mathrm{~d}$ 时,所有柱样上覆水中的 DMS 浓度均开始下降,这也与嗅味记录中湖泛柱 样在实验后期臭味减轻的现象相一致.
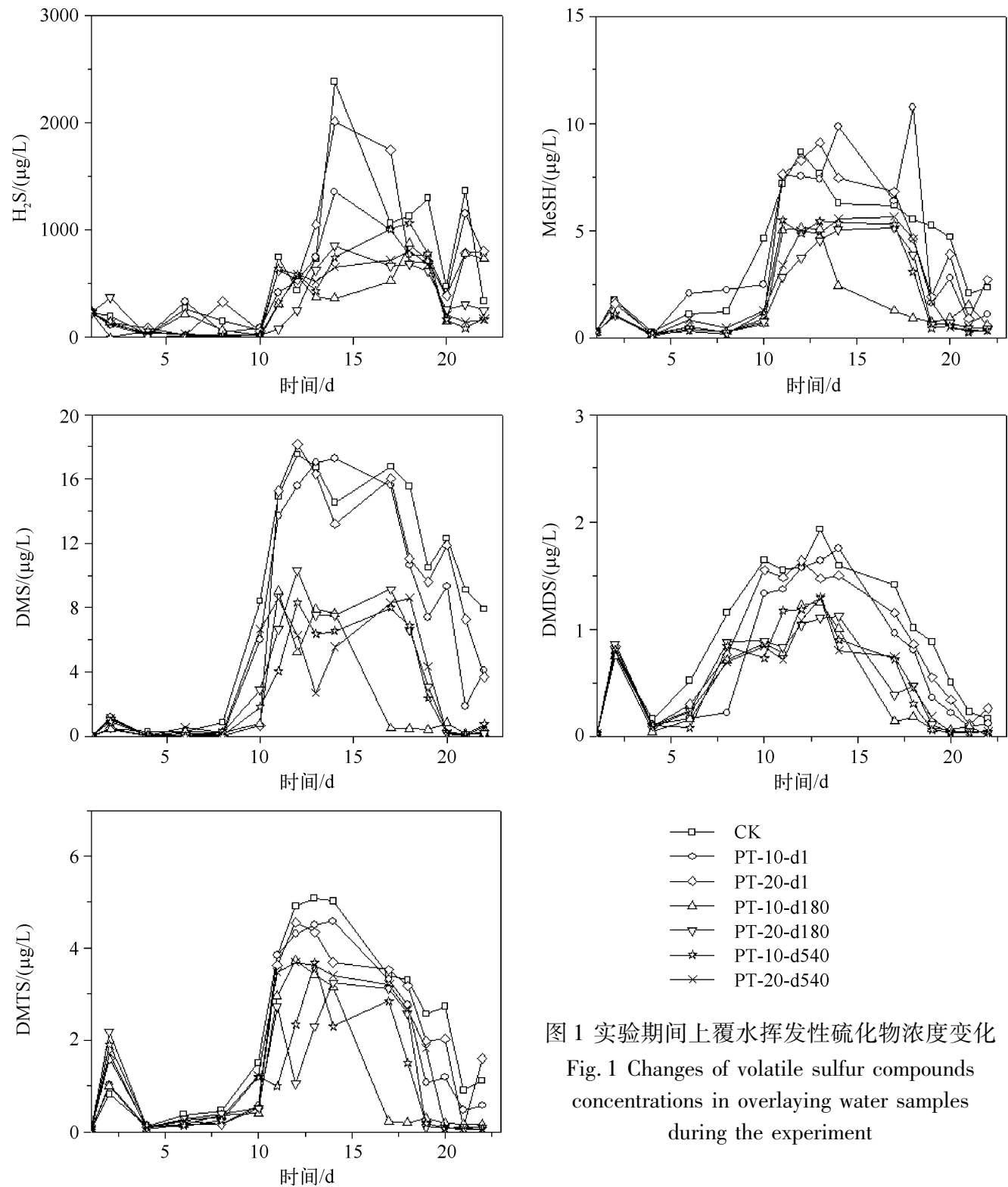

图 1 实验期间上覆水挥发性硫化物浓度变化

Fig. 1 Changes of volatile sulfur compounds concentrations in overlaying water samples during the experiment

柱样上覆水中 DMDS 和 DMTS 浓度的变化趋势较为一致,在实验第 $2 \mathrm{~d}$ 投加了沥干藻体之后, DMDS 和 DMTS 浓度均有较大幅度提高, 但是很快下降, 这可能是投加的藻体很快被分解所致, 两者分别在第 $6 \mathrm{~d}$ 和 第 $11 \mathrm{~d}$ 时浓度大幅提高. CK、PT-10-d1 和 PT-20-d1 柱样上覆水中的 DMDS 和 DMTS 浓度要高于其它 4 个柱 
样. 在实验后期, 所有柱样上覆水中的 DMDS 和 DMTS 浓度均开始下降, 没有发生湖泛的 4 根柱样上覆水的 DMDS 和 DMTS 浓度恢复到实验初始水平, 而发生湖泛的 CK、PT-10-d1 和 PT-20-d1 柱样的 DMDS 和 DMTS 浓度要高于自身的初始水平,说明湖泛明显提高了水体挥发性硫化物的浓度.

$\mathrm{H}_{2} \mathrm{~S}$ 是好氧水体及其沉积物中痕量挥发性硫化物的主要前驱物, $\mathrm{H}_{2} \mathrm{~S}$ 被甲基化后生成 MeSH, 而 MeSH 进一步甲基化生成 DMS, $\mathrm{H}_{2} \mathrm{~S}$ 还可以通过生物氧化过程转化为无机多硫化物, 然后经过生物化学途径被某 些能作为甲基供体的物质如腐殖质中含甲基基团物质、含甲氧基的芳香族化合物甲基化生成 DMDS、DMTS 等 ${ }^{[32]}$. Zhang 等对太湖湖泛的研究则认为水体臭味的主要来源是甲硫醇、二甲基硫醚、二甲基二硫化物和二 甲基三硫化物等挥发性硫化物. 生活污水中的硫跟藻华中的硫不是一个数量级的, 前者不是主要来源, 臭味中 的硫主要来源于厌氧环境下沉积物释放到水相中的硫化物 ${ }^{[6]}$. 通过对臭味指标的记录和对挥发性硫化物浓度 的监测, 认为挥发性硫化物浓度与湖泛发生中臭味的出现和程度变化情况有较强的关联性, 其中又以 $\mathrm{H}_{2} \mathrm{~S}$ 浓
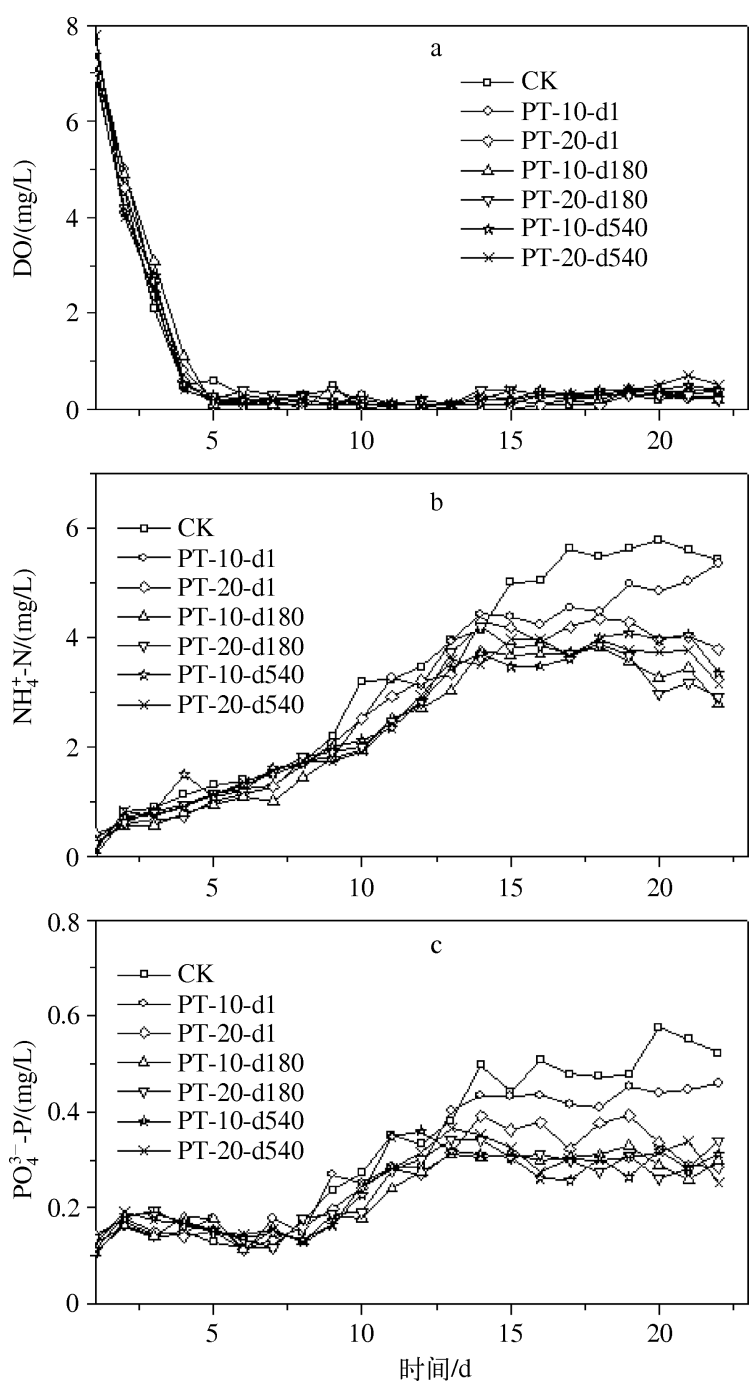

图 2 实验期间上覆水中 $\mathrm{DO}(\mathrm{a}) 、 \mathrm{NH}_{4}^{+}-\mathrm{N}(\mathrm{b})$ 和 $\mathrm{PO}_{4}^{3-}-\mathrm{P}(\mathrm{c})$ 浓度变化

Fig. 2 Changes of dissolved oxygen( a $), \mathrm{NH}_{4}^{+}-\mathrm{N}(\mathrm{b})$ and $\mathrm{PO}_{4}^{3-}-\mathrm{P}(\mathrm{c})$ concentrations during the experiment
度与臭味记录值的对应性较高, 因此, 在模拟实 验中人为所记录到的臭味以 $\mathrm{H}_{2} \mathrm{~S}$ 为主, 挥发性 硫化物浓度能在一定程度上表征湖泛发生的 过程和程度.

\section{3 理化性质变化}

2.3.1 上覆水理化性质变化一般认为湖泛 的发生使有机物分解, 降低底部水体的 DO 浓 度,造成局部厌氧环境, 表层污染程度较高的 沉积物在大量沉降的藻体和厌氧环境共同作 用下形成湖泛现象. 实验开始时, 各柱样上覆 水中的 DO 浓度接近 $8 \mathrm{mg} / \mathrm{L}$, 接近实验温度下 的饱和 DO 浓度, 但是在投加藻类之后, DO 浓 度直线下降, 当水体 DO 浓度小于 $2 \mathrm{mg} / \mathrm{L}$ 时, 水体为厌氧状态, 而投加的藻类使各柱样上覆 水中 DO 浓度在第 $4 \mathrm{~d}$ 时降至接近 0 , 此后水 体的 DO 浓度也一直保持在 $0 \sim 1 \mathrm{mg} / \mathrm{L}$ 之间 (图 2a).

刘国锋等在模拟湖泛实验中也发现投加 藻体之后, 沉积物柱样上覆水的 DO 浓度在很 短时间内就下降到接近 0 的水平 ${ }^{[16]}$, 即使在 模拟湖泛结束后, 没有形成湖泛的柱样上覆水 体中的 DO 浓度也不超过 $1 \mathrm{mg} / \mathrm{L}^{[9]}$. 因此, 湖 泛模拟实验中上覆水体中的 DO 浓度受到投 加藻体的影响, 大量死亡的藻体腐烂分解导致 水体中 DO 浓度迅速下降到厌氧状态.

在实验第 $2 \mathrm{~d}$ 投加藻体之后, 上覆水中 $\mathrm{NH}_{4}^{+}-\mathrm{N}$ 浓度开始逐渐增加, 在第 $10 \mathrm{~d}$ 时, CK、 PT-10-d1 和 PT-20-d1 柱样上覆水中的 $\mathrm{NH}_{4}^{+}-\mathrm{N}$ 浓度迅速增加, 在第 $14 \mathrm{~d}$ 已经明显大于未发 生湖泛的柱样 (图 2b). 相关研究表明, 湖泛中 $\mathrm{NH}_{4}^{+}-\mathrm{N}$ 浓度的提高是藻体腐烂和沉积物释放 共同作用的结果, CK 组没有经过翻耕处理, 底 层上覆水和表层间隙水中的 $\mathrm{TN}$ 和 $\mathrm{NH}_{4}^{+}-\mathrm{N}$ 浓 度均处于较高水平, 而 PT-10-d1 柱样则由于 
刚翻耕 $1 \mathrm{~d}$, 翻耕 $10 \mathrm{~cm}$ 深度的沉积物在翻耕 $1 \mathrm{~d}$ 之后表层间隙水 $\mathrm{NH}_{4}^{+}-\mathrm{N}$ 浓度较高, 释放速率也较大.

在整个实验周期内所有柱样上覆水中的 $\mathrm{PO}_{4}^{3-}-\mathrm{P}$ 浓度变化 (图 2c) 表明, 实验前期, 各柱样上覆水中 $\mathrm{PO}_{4}^{3-}-\mathrm{P}$ 浓度趋势比较平稳, 从第 $9 \mathrm{~d}$ 开始, 各柱样上覆水中 $\mathrm{PO}_{4}^{3-}-\mathrm{P}$ 浓度开始增加, 其中发生湖泛的柱样 CK、PT-10-d1 和 PT-20-d1 增加比较迅速, 各柱样上覆水 $\mathrm{PO}_{4}^{3-}-\mathrm{P}$ 浓度在第 $13 \mathrm{~d}$ 开始出现分化, 其中发生湖泛 的柱样 $\mathrm{PO}_{4}^{3-}-\mathrm{P}$ 浓度继续保持增加趋势, 而未发生湖泛的柱样上覆水中的 $\mathrm{PO}_{4}^{3-}-\mathrm{P}$ 浓度开始下降, 并在第 $16 \mathrm{~d}$ 开始保持稳定. PT-20-d1 柱样 $\mathrm{PO}_{4}^{3-}-\mathrm{P}$ 浓度在实验后期也开始下降,在实验结束时与其它未发生湖泛的柱样 上覆水的 $\mathrm{PO}_{4}^{3-}-\mathrm{P}$ 浓度保持在同一水平.

沉积物中的磷主要以 Fe-P 的形式存在 ${ }^{[33]}$, 在实验中, 藻类投加后, 底部水体中的 DO 浓度不断降低, 在 此区域的氧化还原电位值也会大幅下降,在还原态环境时, 沉积物中被 $\mathrm{Fe}^{3+}$ 所绑定的磷会因为铁被还原而 重新释放到水体中 ${ }^{[34]}$. 而对沉积物的疏浚等较极端的物理扰动会在新生沉积物表层形成一层较薄的氧化 层, 会阻止沉积物向上覆水释放 $\mathrm{PO}_{4}^{3-}-\mathrm{P}$, 且翻耕后沉积物新生表层的 $\mathrm{TP}$ 浓度降低, 因此翻耕处理的沉积物 释放 $\mathrm{PO}_{4}^{3-}-\mathrm{P}$ 的潜力较低 ${ }^{[35-36]}$.

2.3.2 沉积物理化性质变化 (图 3) PT-10-d1 和 PT-20-d1 柱样的表层含水率要低于对照和其它翻耕时间较 长的柱样, 翻耕 $180 \mathrm{~d}$ 的 2 根柱样的含水率高于翻耕 $1 \mathrm{~d}$ 处理组, 但低于对照, PT-10-d180 和 PT-20-d180 柱 样的表层含水率分别为 $58.85 \%$ 和 $58.65 \%$, 翻耕 $540 \mathrm{~d}$ 的处理组柱样表层含水率与对照组相近. 翻耕处理 组的沉积物柱样在不同翻耕后时长和不同翻耕深度下表层沉积物处的 LOI 基本保持在一个水平上,其中 PT-20-d1柱样的 LOI 要比其它翻耕处理的略低, 表层 LOI 为 $4.84 \%$, 其它翻耕处理的沉积物柱样表层 LOI 在 $5.24 \% \sim 5.42 \%$ 之间,而对照组的沉积物表层 LOI 高达 $6.67 \%$, 说明翻耕处理对沉积物的表层 LOI 有较好的 控制, 对照组的表层有机质含量要高于翻耕处理组.

沉积物的 TN 和 TP 含量的垂向剖面比较相似,均为表层沉积物对照组要高于翻耕处理组, 其中又以 TP 含 量的垂向剖面最为明显, CK 柱样的表层沉积物 TP 含量高达 $630.44 \mathrm{mg} / \mathrm{kg}$, 而在翻耕处理组中表层沉积物 TP 含量最高的 PT-20-d180 柱样也仅有 $419.28 \mathrm{mg} / \mathrm{kg}$, 其余柱样沉积物表层 TP 含量则分布在 $288.55 \sim 343.42$ $\mathrm{mg} / \mathrm{kg}$ 之间. 由此可见,在翻耕处理后,翻耕处理组的沉积物表层 LOI、TP 和 TN 含量等会大幅下降,物理修 复手段可以形成污染物含量低的表层沉积物.

实验表明, CK 柱样在模拟湖泛中发生湖泛现象,而翻耕处理的 PT-10-d1 和 PT-20-d1 柱样在实验周期中 也发生了湖泛现象,但是在嗅味和水色以及挥发性硫化物浓度指标上均轻于 CK 柱样,这 2 根柱样发生湖泛 可能是由于在翻耕后较短时期内间隙水中氧化还原条件导致上覆水中 $\mathrm{NH}_{4}^{+}-\mathrm{N}$ 和 $\mathrm{PO}_{4}^{3-}-\mathrm{P}$ 浓度在翻耕后的 短期内处于较高水平, 因此翻耕 $1 \mathrm{~d}$ 后处理组沉积物柱样在模拟实验中也发生了湖泛,但是由于翻耕后表层 沉积物的 TP、TN 含量和 LOI 降低, 即沉积物释放污染物质的潜力会大大降低, 因此 7 根柱样在投加相同重 量的沥干藻体之后, PT-10-d1 和 PT-20-d1 柱样无论是在湖泛时间还是黑臭程度上都要轻于 CK 柱样, 只要在 实际翻耕操作的时候避开湖泛易暴发的时间,翻耕将是非常经济的预控湖泛的有效手段.

\section{3 结论}

1) 对污染较严重的表层沉积物翻耕可以形成新的沉积物一水界面,新生的表层沉积物和其间隙水营养 盐含量均较低, 可以有效改善湖泊表层底泥和底部水体污染状况, 是一种有效治理湖泊内源污染的手段,该 方法具有操作简单、易实施、成本低的特点.

2) 表层底泥翻耕可以有效控制湖泛的发生和程度. 在模拟实验中,对照组和模拟翻耕后 $1 \mathrm{~d}$ 不同深度 的柱样出现黑臭现象,但翻耕后 $1 \mathrm{~d}$ 的处理组无论是在湖泛发生的持续时间上还是黑臭程度上均远远轻于 对照组,翻耕后 $180 \mathrm{~d}$ 和 $540 \mathrm{~d}$ 的处理在模拟实验中未发生湖泛.

3 ) 模拟实验的嗅觉记录中所有柱样上覆水的臭味变化与挥发性硫化物浓度变化具有较高的一致性, 挥 发性硫化物尤其是 $\mathrm{H}_{2} \mathrm{~S}$ 浓度的升高, 是实验中发生湖泛水体产生臭味的主要原因.

4) 翻耕对于预控藻源性湖泛的发生有较好的作用,在湖泛易发时段 (5-7 月) 之前, 提前进行翻耕作 业, 将会有效控制湖泛的发生. 因此科学地管理翻耕施工时间 (如在秋、冬季施工) , 将对易发区藻源性湖泛 起到有效预控作用. 本研究采用的 $10 \mathrm{~cm}$ 和 $20 \mathrm{~cm}$ 模拟翻耕深度对控制湖泛的效果差异不明显, 因此从工程 

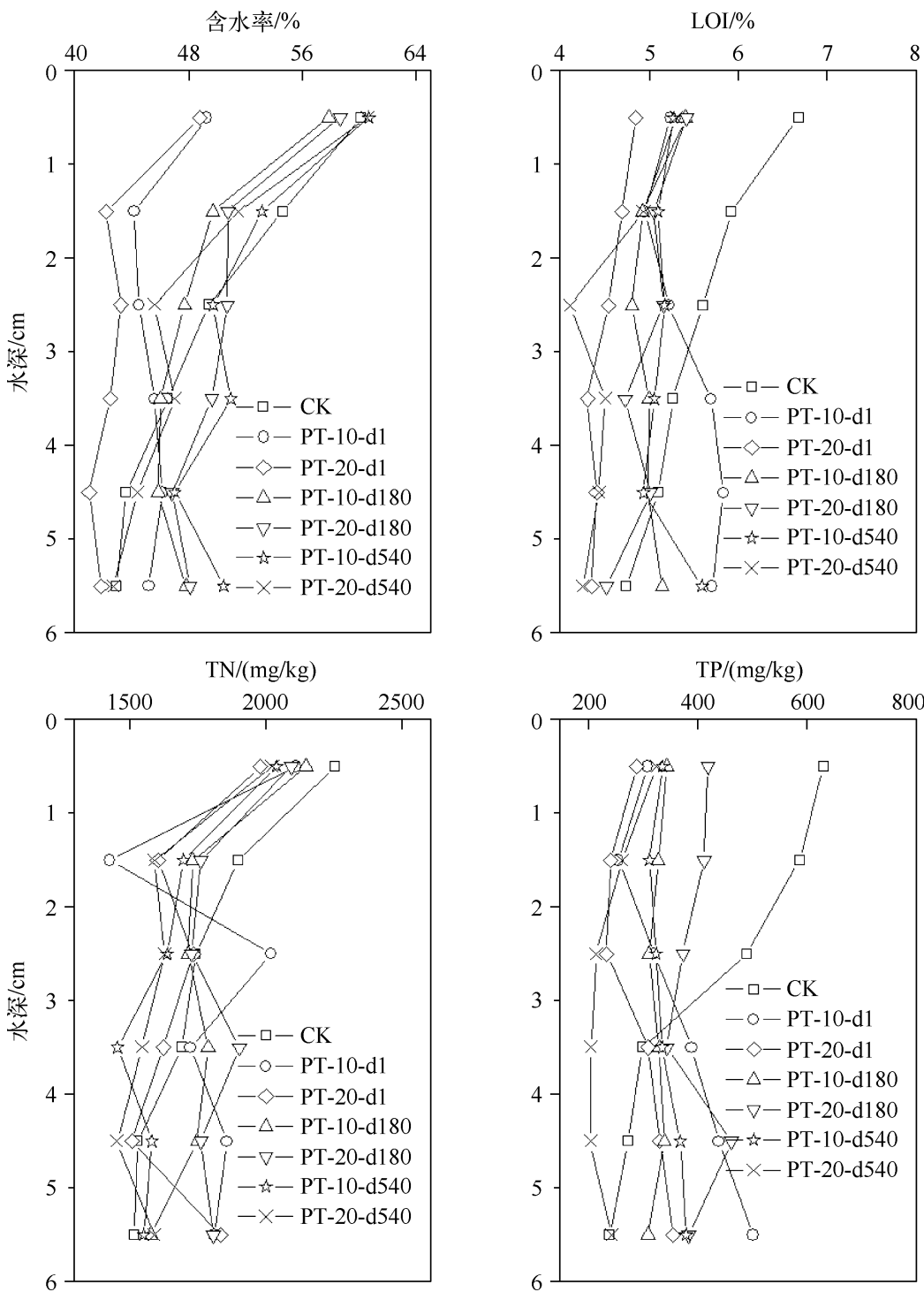

图 3 沉积物柱样理化性质

Fig. 3 Vertical profiles of physicochemical properties in the sediments

经济性考虑, 还可以在以后的工作中继续研究小于 $10 \mathrm{~cm}$ 的适合太湖底泥翻耕的深度.

\section{4 参考文献}

[ 1 ] 陆桂华,马 倩. 太湖水域“湖泛”及其成因研究. 水科学进展,2009,20(3):438-442.

[ 2 ] Yang M, Yu JW, Li ZL et al. Taihu Lake not to blame for Wuxi's woes. Science, 2008, 319(5860) : 158.

[ 3 ] Qin B, Zhu G, Gao G et al. A drinking water crisis in Lake Taihu, China: Linkage to climatic variability and lake management. Environmental Management, 2010, 45(1): 105-112.

[4] 盛 东,徐兆安,高 怡. 太湖湖区“黑水团”成因及危害分析. 水资源保护,2010,26(3):41-44,52.

[5] 刘国锋,何 俊,范成新等. 藻源性黑水团环境效应: 对水-沉积物界面处 $\mathrm{Fe} 、 \mathrm{Mn} 、 \mathrm{~S}$ 循环影响. 环境科学, 2010,31 (11) :2652-2660.

[ 6 ] Zhang XJ, Chen C, Ding JQ et al. The 2007 water crisis in Wuxi, China: Analysis of the origin. Journal of Hazardous Materials, 2010, 182(1/2/3): 130-135. 
[ 7 ] 孔繁翔, 胡维平, 谷孝鸿等. 太湖梅梁湾 2007 年蓝藻水华形成及取水口污水团成因分析与应急措施建议. 湖泊科 学, 2007,19(4):357-358

［8］陆桂华,马 倩. 2009 年太湖水域“湖泛”监测与分析. 湖泊科学,2010,22(4):481-487.

[ 9 ] He W, Shang J, Lu X et al. Effects of sludge dredging on the prevention and control of algae-caused black bloom in Taihu Lake, China. Journal of Environmental Sciences, 2013, 25(3) : 430-440.

[10］马绍赛. 乳山湾菲律宾蛤仔养殖滩涂老化修复实验研究. 海洋水产研究,2005,26(2):59-61.

[11] 陈聚法,张东杰,宋建中等. 乳山湾䍀蛏养殖老化滩涂的修复研究. 海洋水产研究,2005,26(5):57-61.

[12] 何 伟. 湖泊表层沉积物翻耕对内源控制及底栖生物影响研究 [ 学位论文]. 南京: 中国科学院南京地理与湖泊研 究所,2013.

[13] 古小治,王 强,张 雷等. 物理改良对湖泊沉积物和间隙水特征的影响. 中国环境科学,2010,30(2):256-262.

[14] Gu XZ, Chen KN, Huang W et al. Preliminary application of a novel and cost-effective in-site technology in compacted lakeshore sediments for wetland restoration. Ecological Engineering, 2012, 44: 290-297.

[15] 王成林,张 咏,张宁红等. 太湖藻源性“湖泛”形成机制的气象因素分析. 环境科学, 2011,32(2):401-408.

[16] 刘国锋,申秋实,张 雷等. 藻源性黑水团环境效应: 对水-沉积物界面氮磷变化的驱动作用. 环境科学, 2010,31 (12) :2917-2924.

[17] 申秋实,邵世光,王兆德等. 太湖月亮湾湖泛发生过程模拟及水土物化性质的响应. 水科学进展,2011,22(5): 710-719.

[18］王雯雯,姜 霞,王书航等. 太湖竺山湾污染底泥环保疏浚深度的推算. 中国环境科学,2011,31(6): 1013-1018.

[19］范成新,刘元波,陈荷生.太湖底泥蓄积量估算及分布特征探讨.上海环境科学,2000,19(2):72-75.

[20] Rusch A, Töpken H, Böttcher ME et al. Recovery from black spots: results of a loading experiment in the Wadden Sea. Journal of Sea Research, 1998, 40(3/4) : 205-219.

[21] 卢 信,冯紫绝,商景阁等. 不同有机基质诱发的水体黑臭及主要致臭物(VOSCs)产生机制研究. 环境科学,2012, $33(9): 3152-3159$.

[22] Lu X, Fan C, Shang J et al. Headspace solid-phase microextraction for the determination of volatile sulfur compounds in odorous hyper-eutrophic freshwater lakes using gas chromatography with flame photometric detection. Microchemical Journal, 2012, 104(9): 26-32.

[23] 魏复盛. 水和废水监测分析方法:第 4 版. 北京: 中国科学出版社,2002:254-257,435-438.

[24] Murphy J, Riley JP. A modified single solution method for determination of phosphate in natural waters. Analytica Chimica Acta, 1962, 26(1) : 31-36.

[25] 钟继承,刘国锋,范成新等. 湖泊底泥疏浚环境效应: I . 内源磷释放控制作用. 湖泊科学, 2009,21(1):84-93.

[26] Ruban V, Lopez-Sanchez JF, Pardo P et al. Selection and evaluation of sequential extraction procedures for the determination of phosphorus forms in lake sediment. Journal of Environmental Monitoring, 1999, 1(1) : 51-56.

[27] Stahl JB. Black water and 2 peculiar types of stratification in an organically loaded strip-mine lake. Water Research, 1979, $13(5)$ : $467-471$.

[28] Duval B, Ludlam SD. The black water chemocline of meromictic Lower Mystic Lake, Massachusetts, USA. International Review of Hydrobiology, 2001, 86(2) : 165-181.

[29] Freitag TE, Klenke T, Krumbein WE et al. Effect of anoxia and high sulphide concentrations on heterotrophic microbial communities in reduced surface sediments(Black Spots) in sandy intertidal flats of the German Wadden Sea. Fems Microbiology Ecology, 2003 , 44(3) : 291-301.

[30] Neira C, Rackemann M. Black spots produced by buried macroalgae in intertidal sandy sediments of the Wadden Sea: Effects on the meiobenthos. Journal of Sea Research, 1996, 36(3/4) : 153-170.

[31] Bottcher ME, Oelschlager B, Hopner T et al. Sulfate reduction related to the early diagenetic degradation of organic matter and "black spot" formation in tidal sandflats of the German Wadden Sea ( southern North Sea): stable isotope (C-13, S-34, O-18) and other geochemical results. Organic Geochemistry, 1998, 29(5/6/7) : 1517-1530.

[32] 卢 信. 藻源性湖泛主要致臭物(VSCs) 形成机制及底泥疏浚影响研究 [学位论文]. 南京: 中国科学院南京地理与 湖泊研究所,2012.

[33] Graca B, Burska D, Matuszewska K. The impact of dredging deep pits on organic matter decomposition in sediments. Water, Air, and Soil Pollution, 2004, 158(1) : 237-259.

[34] Li QM, Zhang W, Wang XX et al. Phosphorus in interstitial water induced by redox potential in sediment of Dianchi Lake, China. Pedosphere, 2007, 17 (6) : 739-746.

[35] Zhong JC, You BS, Fan CX et al. Influence of sediment dredging on chemical forms and release of phosphorus. Pedosphere, 2008, 18(1): 34-44.

[36] 余居华,钟继承, 张银龙等. 湖泊疏浚对沉积物再悬浮及磷迁移影响的模拟研究. 环境科学, 2012,33 (10): 3368-3375. 